\title{
Short survey
}

\section{A taxonomy of multi-area state estimation methods}

\author{
Antonio Gómez-Expósito ${ }^{\mathrm{a}, *}$, Antonio de la Villa Jaén ${ }^{\mathrm{a}}$, Catalina Gómez-Quiles ${ }^{\mathrm{a}}$, \\ Patricia Rousseaux ${ }^{b}$, Thierry Van Cutsem ${ }^{\text {b, } 1}$ \\ a Department of Electrical Engineering, University of Seville, Camino de los Descubrimientos $s / n, 41092$ Seville, Spain \\ ${ }^{\mathrm{b}}$ Department of Electrical Engineering and Computer Science (Montefiore Institute) of the University of Liège, Sart Tilman B37, B-4000 Liège, Belgium
}

\section{A R T I C L E I N F O}

\section{Article history:}

Received 21 July 2010

Received in revised form 27 October 2010

Accepted 16 November 2010

Available online 19 December 2010

\section{Keywords:}

Multi-area state estimation

Hierarchical state estimation

Distributed state estimation

Factorized state estimation

\begin{abstract}
A B S T R A C T
This paper presents a critical review of the state of the art in multi-area state estimation (MASE) methods, which are currently gaining renewed interest due to their capability of properly tracking multi-TSO transactions and accommodating highly redundant information systems. Based on several classification criteria, a taxonomy of MASE methods is first proposed. Two main categories, namely two-step or hierarchical versus decentralized, are identified. Then, for each class of methods, the resulting model structure and area interactions are discussed and a brief presentation is made of a selected subset of references.
\end{abstract}

(c) 2010 Elsevier B.V. All rights reserved.

\section{Introduction}

State estimators (SE) determine the most likely state of a power system from sets of measurements which are captured remotely at substations and collected periodically by SCADA systems via remote terminal units (RTU). The information provided by the SE is crucial in nowadays energy management systems (EMS), where a diversity of applications dealing with the economic and secure operation of transmission networks rely on accurate and continuously updated snapshots of the system. The new regulatory paradigm arisen in the last decade has even stressed the importance of the SE tool, in an open-access context in which many more transactions on much more congested networks have to be properly tracked.

Research on multi-area state estimation (MASE) can be traced back to the late 1970s, shortly after state estimators started being put into service. It addressed the problem of performing efficient state estimation on large power systems, with the twofold motivation of gaining computing time (under the then-limited computational resources) and exploiting the fact that real-time measurements are gathered within areas by the various control centers distributed over the grid. MASE relies on some kinds of decomposition-coordination scheme, taking advantage of the usu-

\footnotetext{
* Corresponding author.

E-mail address: age@us.es (A. Gómez-Expósito).

$1 \mathrm{He}$ is with the Fund for Scientific Research - FNRS.
}

ally weaker geographical or measurement coupling among areas, in combination with well-established solution methods.

MASE has regained interest over the last decade owing to projects of having central entities monitoring large interconnected systems. This is the case, for instance, of Independent System or Regional Transmission Operators in the U.S. [1], while projects of supra-national centers monitoring the European grid start becoming a reality (e.g. Coreso [2]). Furthermore, large-scale incidents experienced over the recent years have stressed the need for a better real-time visibility of the operating state of the grid well beyond the extent covered by the state estimator of a single country or company. For reliability, computational efficiency and model maintenance reasons, it does not sound reasonable to collect and process the huge set of data of those large grids at a single place. Hence, the idea of MASE.

This paper proposes a taxonomy of MASE methods, offering a unifying description of a relatively large number of works devoted to the subject, and probably a few more to come, with the advances in phasor measurement technology. It significantly enhances and updates the only known survey on the topic, published early in the eighties [3]. Due to space limitations, it has not been possible to exhaustively include all publications in the comparative analysis. Neither does the paper deal with the interesting related problems of bad data identification and observability analysis.

Other applications of parallel and distributed processing to power systems are quoted in [4].

The structure of this paper is as follows. Section 2 briefly reviews the conventional nonlinear SE formulation, Section 3 lists the most 
important desirable features of MASE and Section 4 defines the notation adopted in the paper. The criteria used to classify MASE methods are defined in Section 5. A unified description of the hierarchical and the decentralized approaches is presented in Sections 6 and 8, respectively, while the corresponding literature surveys are given in Sections 7 and 9. Section 10 offers the conclusions.

\section{Background on state estimation}

The SE relies on the following measurement equation [5]:

$z=h(x)+e$

where:

$x$ is the state vector to be estimated (size $n$ ), $z$ is the known measurement vector (size $m>n$ ), $h$ is the vector of functions, usually nonlinear, relating error free measurements to the state variables,

$e$ is the vector of measurement errors, customarily assumed to have a Normal distribution with zero mean and known covariance matrix $R$. When errors are independent $R$ is a diagonal matrix with values $\sigma_{i}^{2}$, where $\sigma_{i}$ is the standard deviation of the error associated with measurement $i$.

In conventional bus-branch SE models the state vector is composed of voltage magnitudes and phase angles, whereas the measurement vector typically comprises power injections, branch power flows and voltage magnitudes. Recently, the availability of synchro-phasors (PMUs) has made it possible to incorporate phase angle measurements into the SE process.

The weighted least squares (WLS) estimator minimizes:

$J=\sum_{i=1}^{m} W_{i} r_{i}^{2}$

where:

$r_{i}=z_{i}-h_{i}(\hat{x})$ is the measurement residual,

$\hat{x}$ is the estimated state vector, and

$W_{i}$ is the respective weighting coefficient.

The state estimate can be obtained by iteratively solving the so-called Normal equations:

$G_{k} \Delta x_{k}=H_{k}^{T} W\left[z-h\left(x_{k}\right)\right]$

where:

$H_{k}=\partial h / \partial x$ is the Jacobian evaluated at $x=x_{k}$,

$G_{k}=H_{k}^{T} W H_{k}$ is the gain matrix,

$W=\operatorname{diag}\left(W_{i}\right)$ is the weighting matrix,

$\Delta x_{k}=x_{k+1}-x_{k}, k$ being the iteration counter.

Iterations are terminated when an appropriate tolerance is reached on $\Delta x_{k}$. The covariance of the estimate is:

$\operatorname{Cov}(\hat{x})=G_{k}^{-1}$

provided the covariance $\sigma_{i}^{2}$ is used as weight $W_{i}$.

Then, the bad data processing function, aimed at detecting, identifying and eliminating bad analog measurements, is activated. This usually relies on the normalized residual test [6].

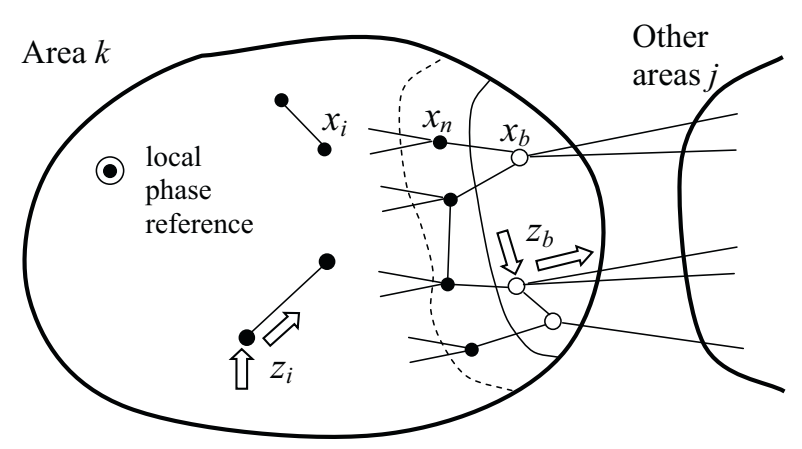

Fig. 1. Classification of measurements and state variables.

\section{Desirable features of MASE}

In principle, processing the whole measurement set in a single WLS estimator provides the "optimal" estimate. This conventional scheme is referred to as "integrated" in the sequel. This section enumerates the most desirable features that a MASE should exhibit compared to the integrated scheme.

- Robustness: the capability to converge to an acceptable solution under a wide range of circumstances (topology, measurement configuration, the presence of large bad data, etc.). Problem decomposition may affect convergence properties of MASEs.

- Accuracy: the estimate has to be accurate enough for operating purposes. Ideally, it should be the same as that with the integrated scheme. In practice, optimality is not a finality per se: other advantages gained out from decomposed approaches can be considered as more important, provided accuracy remains acceptable of course.

- High computational efficiency: increased speed is one of the expected attractive features of MASE, provided that the algorithmic complexity linked to the decomposition-coordination remains limited.

- Amount of data exchange: information exchange between processors should be kept as low as possible in order to avoid possible time delays and thus an increase in the estimator response time and/or require a larger communication bandwidth. Attention should also be paid to not having many measurements shared by more than one computer, which increases the complexity of the data acquisition process.

- Bad data analysis: the bad data rejection capability should be preserved, more specifically in the proximity of area boundaries. The possibility to easily compute the normalized residuals is another important issue.

\section{Nomenclature}

This section provides the common definitions and notation that will serve to understand the diverse MASE procedures described below, some of them differing only in subtle details, which are frequently hidden by the particular jargon used in the original publication.

Consider an arbitrary area $k$, directly connected to other areas through tie-lines, as shown in Fig. 1. The state variables of area $k$ can be classified as follows:

- $x_{i k}$ : internal variables, associated with buses which are not terminals of tie-lines;

- $x_{b k}$ : border variables, associated with terminal buses of tie-lines;

- $x_{n k}$ : subset of variables in $x_{i k}$ associated with first neighbors of border buses. In certain cases, this subset may contain second and, eventually, deeper neighbors of border buses, depending 


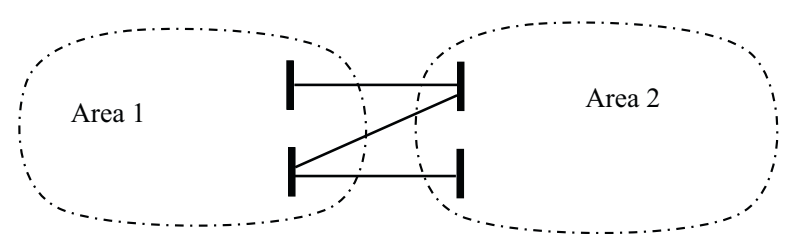

Fig. 2. Non-overlapping areas.

on the amount of information that adjacent areas are willing to exchange.

The phase angles of buses in area $k$ (internal and border buses) are referred for convenience to that of an arbitrary local reference bus. Such relative phase angles are involved in the expression of any power flowing in internal branches. However, power flows through tie-lines, as well as power injections at tie-line terminal buses, involving buses of different areas, require that absolute phase angles be used. Therefore, for a system comprising $A$ interconnected areas, an additional set of variables, termed $u$, is introduced containing the phase angle differences of $A-1$ local references with respect to the remaining one, chosen as the absolute reference.

To distinguish between both references, a vector $x$ will denote state variables referred to the global phase reference, whereas a vector $y$ will be used when voltage phase angles refer to their respective local reference.

A similar classification of available measurements can be made, again for any given area $k$, as illustrated also in Fig. 1:

- $z_{i k}$ : internal measurements, exclusively related with variables $x_{i k}$ (or $y_{i k}$ ) and $x_{b k}$ (or $y_{b k}$ ) in area $k$;

- $z_{b k}$ : border measurements, functions of $x_{b k}$ and the vectors $x_{b j}$ of other areas $(j \neq k)$, and possibly $x_{n k}$.

\section{Classification criteria of MASE}

MASE methods can be classified and discussed according to a variety of criteria detailed hereafter.

\subsection{Area overlapping level}

MASE procedures are based on system decomposition into interconnected areas. Depending on the coordination strategy adopted to reconcile local estimates, the degree of overlapping, i.e. the number of buses and/or branches in common between two adjacent areas, may range from zero to several layers of border buses and associated branches, as explained below:

(1) Non-overlapping areas have no bus and no branch in common; they are connected by tie-lines ending at border buses (see Fig. 2). Those tie-lines define the interconnection area.

(2) Border-bus overlapping areas are adjacent areas overlapping over just one layer of border buses (see Fig. 3); there is no tie-line connecting two areas. This situation can be artificially created from the previous case, defining a virtual border bus at the mid-point of each tie-line and extending each area up to

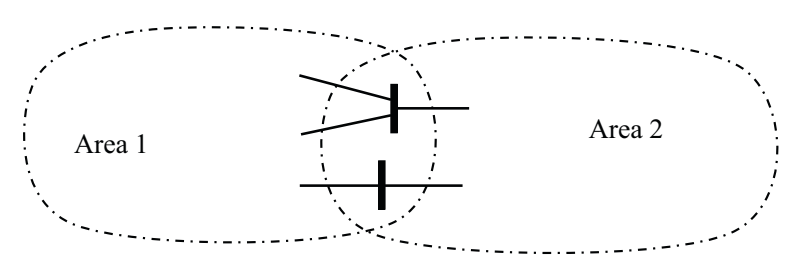

Fig. 3. Border-bus overlapping areas.

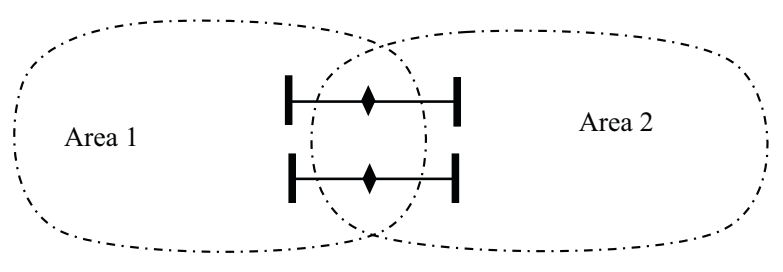

Fig. 4. Mid-point virtual bus overlapping areas.

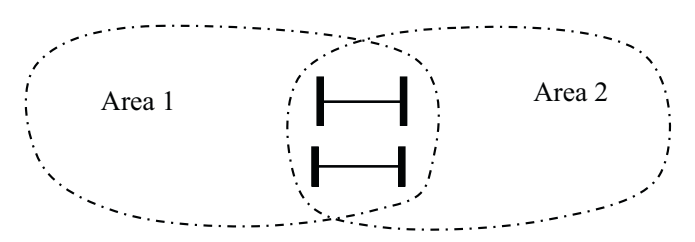

Fig. 5. Tie-line overlapping areas.

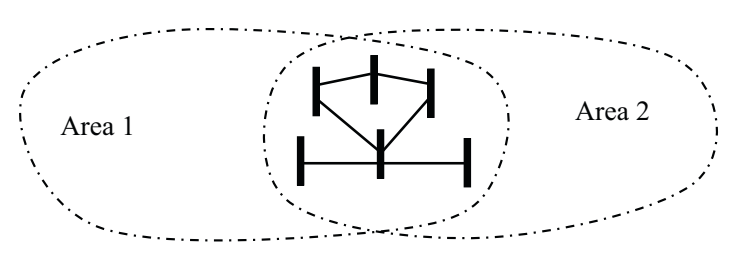

Fig. 6. Extended overlapping areas.

this new bus (see Fig. 4). This particular decomposition will be referred to as "mid-point virtual bus overlapping areas". By so doing, one gets rid automatically of boundary injection measurements.

(3) Tie-line overlapping areas share tie-lines and the corresponding border buses (see Fig. 5).

(4) Extended (or deep) overlapping areas share several layers of neighbors of border buses (see Fig. 6).

For classification purposes, the following simplified terminology will be adopted: non-overlapping areas, minimally overlapping areas (item 2) and fully overlapping areas (items 3 and 4).

\subsection{Computing architecture}

Two computer architectures and hardware environments are relevant:

(1) In a hierarchical scheme (see Fig. 7), a master processor distributes the work among slave computers performing local area SE and, subsequently, coordinates the local estimates. In this scheme, slave processors, which can be located remotely (distributed architecture) or at the same physical place (parallel architecture), communicate only with the central computer.

(2) in a decentralized architecture (see Fig. 8), there is no central computer; each local processor communicates only with those processors in charge of neighboring areas, exchanging border

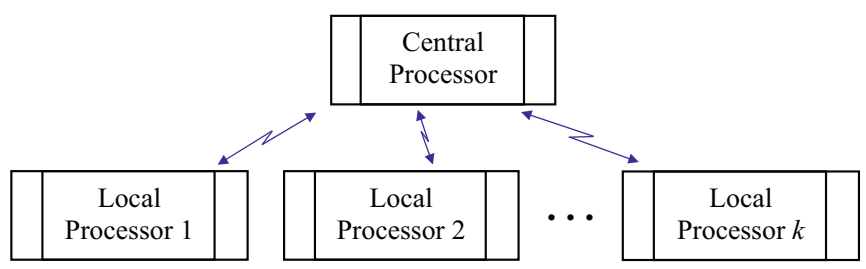

Fig. 7. Hierarchical architecture. 


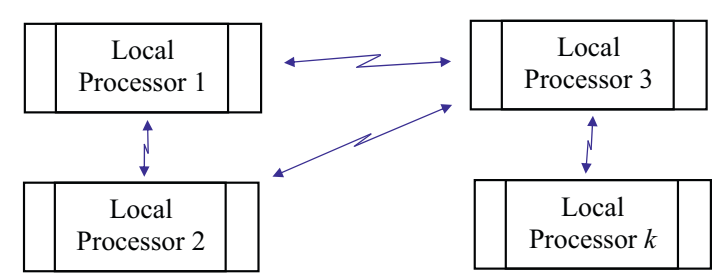

Fig. 8. Decentralized distributed architecture.

information. Usually, the iterative estimation algorithm relying on this architecture does not involve any coordination phase.

\subsection{Coordination scheme}

Depending on the stage at which the local estimates get coordinated, three coordination schemes can be distinguished.

(1) Coordination at the SE level: each area submits its results to the central processor after full convergence of its local SE. Methods relying on this coordination type presents the following essential features:

The central level refines and coordinates local estimates by computing the $u$ variables. When resorting to a single coordination, this strategy leads to suboptimal estimates, but usually of acceptable accuracy; communication bandwidth requirements are kept low by limiting the data exchanges between the central and the local processors; independent SEs allow simple implementation at the local level with minor adaptations to existing SE software packages.

(2) Coordination at the iteration level: results are submitted for coordination after each iteration of the local SE, with the following main features:

Repeated coordinations allow faster convergence to the optimal solution than in the previous scheme; the price to pay is an important increase in data exchange between the central and the local computers or between neighboring areas in the decentralized architecture; generally, it requires more complex code implementations, and does not allow to keep existing SE algorithms.

(3) Hybrid coordination is a compromise between the above two schemes, in which several local iterations are performed before coordination.

\subsection{Measurement synchronization}

When considering only conventional power and voltage magnitude measurements, synchronization between areas may be an issue. Either we look for similar accuracy to that of an integrated scheme or we accept some suboptimality. In the former case, it is important to synchronize the various measurement gatherings so that the hierarchical scheme does not add to the time skew present in conventional data acquisition (e.g., a synchronizing signal could be sent to the various local computers). If suboptimality is accepted, this synchronization requirement can be somewhat relaxed.

Incorporating information provided by PMU provides new possibilities for measurement synchronization. In theory, using a whole set of measurements coming from PMU would allow full synchronization of snapshots captured by all areas. In practice, however, this may be too cumbersome or even impossible.

\subsection{Process synchronization}

Local processors may run in a synchronous or asynchronous manner. In the decentralized architecture, the process is asyn- chronous by nature: each local processor performs iterations at its own pace, using the best information available from its neighbors. In the hierarchical scheme, two synchronization modes are possible:

- when coordination takes place at the SE level, there is no need to impose synchronous local estimations so that each local processor usually runs its SE at its own rate. The central processor always uses the last estimate provided by each area, no matter if those local estimates do not refer to the same time instant. This time skew among local estimates may affect the accuracy of the final solution;

- when coordination takes place at the iteration level, the central computer may (synchronous) or may not (asynchronous) wait for the slowest local processor to complete the current iteration, before coordinating the solution.

\subsection{Solution methodology}

Various general methodologies can be used to solve the decomposition-coordination problem involved in MASE. A large majority of methods rely on the classical WLS formulation. Most hierarchical schemes rely, at both the local and the central levels, on an iterative scheme to solve the Normal equations of concern. In decentralized schemes, with possible coordination at the iteration level, Lagrangian relaxation-based algorithms are usually adopted. Some works also introduce certain heuristics intended to simplify the optimality conditions of the coordination problem. A category of MASE approaches formulate the WLS equations as an optimization problem, usually involving a Lagrangian function explicitly handling constraints imposed by network equations and/or boundary conditions.

Among the above criteria, the one related to computing architecture is adopted to further classify existing MASE methods in two main categories: two-step hierarchical vs. decentralized MASE, respectively. In the following sections, for each category, a short presentation is followed by a literature review.

\section{Two-step hierarchical MASE}

In this section, we consider hierarchical SE methods comprising two main steps: (1) local solutions are obtained at the area level, ignoring totally or partly the information and constraints stemming from neighboring areas; (2) local solutions are coordinated by a central processor in order to cope with the interactions among areas ignored during the first stage. Each step is separately discussed below.

\subsection{First step: local solutions}

During the first stage each area $k$ independently solves a local WLS problem based on the measurement equation:

$z_{k}=h_{k}\left(y_{k}\right)+e_{k}$

where phase angles in $y_{k}$ refer to the local phase reference of area $k$. The components of $y_{k}$ and $z_{k}$ are determined by the overlapping level adopted, as follows:

- Non overlapping:

$$
y_{k}=\left[\begin{array}{c}
y_{i k} \\
y_{b k}
\end{array}\right] ; \quad z_{k}=z_{i k}
$$

By definition, each state variable is estimated only once, within its respective area. Note that tie-line power flows and border injec- 
tions cannot be individually used in this scheme, as they involve external state variables. However, $z_{k}$ may additionally include certain pseudomeasurements, obtained by previously processing raw border measurements. For instance, if the injection at a given border bus, along with all power flows leaving the incident tie-lines, are measured, then a pseudomeasurement representing the net power injection can be added to $z_{k}$.

- Border-bus overlapping:

$y_{k}=\left[\begin{array}{c}y_{i k} \\ y_{b k}\end{array}\right] ; \quad z_{k}=z_{i k}$

The tie-line notion vanishes in this case, as every line fully lies within a single area. Accordingly, all power flow measurements can be handled by their respective local estimator. However, border injections cannot be handled at this level, as they involve external variables. Note that several (at least two) estimates result for the same border variables, each one referred to its own phase reference.

- Tie-line overlapping:

$y_{k}=\left[\begin{array}{c}y_{i k} \\ y_{b k} \\ y_{b j}\end{array}\right] ; \quad z_{k}=\left[\begin{array}{c}z_{i k} \\ z_{b k}\end{array}\right]$

In this case, border injection measurements can be used within their respective area and the possibility exists for tie-line power flow measurements to be used twice. Like in the former case, border state variables are estimated at least twice, each one referred to its own phase reference.

- Extended overlapping:

$y_{k}=\left[\begin{array}{c}y_{i k} \\ y_{b k} \\ y_{b j} \\ y_{n j}\end{array}\right] ; \quad z_{k}=\left[\begin{array}{c}z_{i k} \\ z_{b k} \\ z_{b j}\end{array}\right]$

In this case, the state vector includes the subset $y_{n j}$, which means that border injections of neighboring areas can be also added to $z_{k}$.

In summary, the geographical scope of each local problem determines the set of variables included in the state vector and, consequently, the set of measurements that can be locally handled.

Once the iterative process has converged, the local solution $\tilde{y}_{k}$ is obtained, along with its covariance matrix:

$\operatorname{Cov}\left(\tilde{y}_{k}\right)=G_{k}^{-1}=\left(H_{k}^{T} W_{k} H_{k}\right)^{-1}$

where $H_{k}$ is the Jacobian of $h_{k}$, computed during the last iteration, and $W_{k}$ the respective weighting submatrix.

Note that the estimates $\tilde{y}_{i k}$ of variables sufficiently distant from the border are generally optimal for practical purposes after the local solution process, as the influence of border measurements is negligible. However, the estimates $\tilde{y}_{b k}$ and, to a lesser extent, $\tilde{y}_{n k}$ are suboptimal, unless the border measurements are very accurate.

\subsection{Second step: centralized coordination}

The geographical scope of this step is determined by the overlapping level adopted and the amount of information each area is willing to exchange with its neighbors.

The state vector at this stage comprises at least the border variables corresponding to the $A$ areas, along with the phase reference vector $u$ introduced above, i.e.

$x_{c}=\left[\begin{array}{c}x_{b} \\ u\end{array}\right] \quad$ with $x_{b}=\left[x_{b 1}, \ldots, x_{b k}, \ldots, x_{b A}\right]^{T}$

while the measurement vector at this step includes two components:

$z_{c}=\left[\begin{array}{l}\tilde{y}_{b} \\ z_{b}\end{array}\right]$

where $\tilde{y}_{b}$ is the local estimate of $x_{b}$, with phase angles referred to local buses, and

$z_{b}=\left[z_{b 1}, \ldots, z_{b k}, \ldots, z_{b A}\right]^{T}$

represents the set of border measurements not yet used at the first step. Note that, for each component of the state vector $x_{b}$ two or more estimates may be available in $\tilde{y}_{b}$, which increases the redundancy to estimate the vector $u$.

In the extended overlapping case, or when border injections are to be handled at this stage, first neighbors of border buses are also involved, leading to the augmented state vector:

$x_{c}=\left[\begin{array}{c}x_{b} \\ x_{n} \\ u\end{array}\right] \quad$ with $x_{n}=\left[x_{n 1}, \ldots, x_{n k}, \ldots, x_{n A}\right]^{T}$

In this case, $z_{c}$ should also contain the component $\tilde{y}_{n}$ of the local estimates, to assure observability.

Including second and further neighbors of border buses in the state vector improves the optimality of the solution after the second step, but leads to heavier information exchange and increased computational effort.

The measurement model of the coordination phase is composed of a nonlinear system:

$z_{b k}=h_{c k}\left(x_{c}\right)+e\left(z_{b k}\right), \quad k=1, \ldots, A$

together with a linear one:

$\tilde{y}_{b}=x_{b}-B u+e\left(y_{b}\right)$

where the entries of $B$ are equal to zero for the voltage magnitude components of $x_{b}$ and equal to one for phase angle components.

In the above measurement model the covariance of $e\left(z_{b k}\right)$ is known and that of $e\left(y_{b}\right)$ is contained in the respective submatrix of $\operatorname{Cov}(\tilde{y})$. As this is a dense matrix, it is customary to obtain and retain only its diagonal elements, leading to suboptimal estimates at the coordination step.

The iterative solution of the WLS problem arising from (4) and (5) provides the estimate $\hat{x}_{c}$. Based on this estimate it is theoretically possible to further refine the values of other internal variables, particularly those close enough to the border, but this is seldom considered in the literature.

\section{Literature survey on hierarchical MASE}

Table 1 provides a classification of hierarchical MASE methods, according to the different criteria identified in Section 5.

Due to space limitations only the most representative proposals are briefly presented. For this purpose they are grouped in two separate subsections, depending on whether the coordination phase takes place only once, at the end of the local SE process, or the local solutions are repeatedly coordinated after each iteration.

\subsection{Coordination at the state estimation level}

In 1972, Clements et al. [7] developed a technique relying on border-bus overlapping. After the local estimation processes finish, the coordinating phase reconciles local estimates, which differ at 
Table 1

Classification of hierarchical MASE methods.

\begin{tabular}{|c|c|c|c|c|c|}
\hline Reference, author & Area overl. ${ }^{a}$ & Solut. meth. ${ }^{\mathrm{b}}$ & Estim. state ${ }^{c}$ & Meas. type ${ }^{d}$ & Coord. scheme ${ }^{\mathrm{e}}$ \\
\hline [7], Clements & MO & $\mathrm{NE}$ & Sub & $\mathrm{C}$ & SE \\
\hline [8], Kobayashi & NO & NE & Opt & $\mathrm{C}$ & It \\
\hline [9], Irving & NO & NE-H & Sub & $\mathrm{C}$ & SE \\
\hline [10], Marsh & NO & $\mathrm{NE}-\mathrm{O}$ & Opt & $\mathrm{C}$ & It \\
\hline [11], Van Cutsem & NO & $\mathrm{NE}$ & Sub & $\mathrm{C}$ & SE \\
\hline [12], Wallach & NO & $\mathrm{NE}-\mathrm{H}$ & Sub & $\mathrm{C}$ & SE \\
\hline [13], Brice & NO & NE-R & Opt & $\mathrm{C}$ & It \\
\hline [14], Mukai & NO & NE-R & Opt & $\mathrm{C}$ & It \\
\hline [15], Kurzin & NO & NE-H & Sub & $\mathrm{C}$ & SE \\
\hline [16], Lo & NO & NE-H & Sub & $\mathrm{C}$ & SE \\
\hline [17], Iwamoto & NO & $\mathrm{NE}-\mathrm{O}$ & Sub & $\mathrm{C}$ & It \\
\hline [18], El-Keib & NO & $\mathrm{NE}-\mathrm{H}$ & Sub & $\mathrm{C}$ & SE \\
\hline [19], Ahmed & NO & $\mathrm{NE}-\mathrm{H}$ & Sub & $\mathrm{C}$ & It \\
\hline [20], El-Keib & NO & $\mathrm{O}$ & Opt & $\mathrm{C}$ & It \\
\hline [21], Falcao & MO & NE-H & Sub & $\mathrm{C}$ & SE-It \\
\hline [22], Korres & NO & $\mathrm{NE}$ & Sub & $\mathrm{C}$ & SE \\
\hline [23], Ebrahimian & MO & $\mathrm{NE}-\mathrm{O}$ & Opt & $\mathrm{C}$ & It \\
\hline [24], Aguado & MO & $\mathrm{NE}-\mathrm{O}$ & Opt & $\mathrm{C}$ & It \\
\hline [25], Zhao & FO & $\mathrm{NE}$ & Sub & $\mathrm{P}$ & SE \\
\hline [27], Yan & NO & $\mathrm{NE}-\mathrm{O}$ & Sub & $\mathrm{P}$ & SE \\
\hline [28], Jeffers & FO & NE-H & Sub & $\mathrm{P}$ & SE \\
\hline [29], Jiang & FO & NE-H & Sub & $\mathrm{P}$ & SE \\
\hline [30], Patel & FO & NE & Sub & $\mathrm{P}$ & SE \\
\hline [31], Jiang & NO & $\mathrm{NE}-\mathrm{O}$ & Opt & $\mathrm{P}$ & It \\
\hline [32], Gómez & NO & $\mathrm{NE}$ & Opt & $\mathrm{C}$ & SE \\
\hline [33], Korres & NO & $\mathrm{NE}-\mathrm{O}$ & Opt. & $\mathrm{C}$ & It \\
\hline
\end{tabular}

a NO: non-overlap; MO: minimally overlap; FO: fully overlap.

b NE: Normal equations; R: relaxation; O: optimization; H: heuristic.

c Opt: optimal; Sub: suboptimal.

d C: conventional only; P: considers PMU.

e SE: SE level; It: iteration level.

common buses. Local state vectors are then re-estimated through a non-iterative WLS procedure, using as pseudo-measurements the previous local estimates along with the estimates at common buses obtained from neighboring areas. Power injection measurements at boundary nodes are not taken into account.

In 1977 and 1979, Irving and Sterling [9] proposed to extend the technique of "diakoptics" or network tearing to the state estimation problem. This technique, previously applied to the load flow problem, exploits the nearly block diagonal structure of the Jacobian matrix, diagonal blocks corresponding to non overlapping regions. Links between blocks come from interconnection measurements. Initial local estimates are corrected according to the diakoptics formulae in order to take into account information brought by interconnection measurements. The estimate is supposed to be obtained in only one iteration. As the exact application of diakoptics leads to cumbersome calculations, the authors proposed mathematical simplifications without providing some physical interpretation.

In 1981, Wallach et al. [12] set up a method intended for parallel computation. The original network is divided into disjoint areas, upon the condition that each resulting area is observable and power flow measurements exist at both ends of tie lines. Such power flows are equivalent to power injections from the point of view of the internal area. A slave processor performs the local WLS solution for each area, in a decoupled manner. Then, the master processor simply adjusts the area phase angle references so that all phase angles refer to a single phase origin. For this purpose, a simplistic technique is adopted, consisting of computing the phase angle difference across one of the tie lines linking adjacent areas.

In 1981 also, Van Cutsem et al. [11] developed a two-level estimator based on non-overlapping areas. Only internal measurements are used at the area level, while the coordination level is aimed at estimating tie-line power flows through vector $u$. In addi- tion to tie-line power flow measurements, local estimates at the boundary buses are added as pseudo-measurements to the coordination phase, for which approximate covariances are adopted (diagonal elements of the respective matrix).

In 1983, Kurzin [15] proposed a method conceptually very similar to that of Van Cutsem. The difference lies in the way coordination is carried out. A heuristic approach using arithmetic means is used. The objective is to reduce the size and as a consequence the computing time, of the coordination estimation. From the active power flow measurements through tie lines, the phase angle differences across those lines are estimated. Then, the average of the computed values, in case there are two or more tie lines, is taken as the final estimate.

In the first part of the 1988 paper by Lo et al. [16], six approaches to coordinate the solution of local state estimators (the first five directly derived from the previous works by Van Cutsem et al. and Kurzin) are analytically formulated and reviewed. In the second part, the performance of those algorithms is experimentally tested. A fast decoupled SE formulation is assumed.

In the work of El-Keib et al. in 1990 [18], an extensive review of hierarchical SE methods formerly published is first presented. Then, an improvement to the method of Van Cutsem et al. is proposed regarding the coordination phase. The use of modified injections at boundary buses is suggested, by invoking Kirchhoff's law at those buses. This requires extending the domain of the measurement vector to include the estimates of power flows in lines connecting the boundary buses with internal buses, increasing the redundancy at the upper level and improving the overall results of the state estimator. No tests are provided of this idea.

In 1995, Falcao et al. [21] proposed a two-step solution methodology combining conventional state estimation algorithms with an optimization technique to cope with coupling constraints at the centralized level. Boundary buses belong to adjacent overlapping areas. The number of boundary buses may be kept to a minimum, 
or may incorporate a few extra (internal) buses in order to facilitate bad data processing. It is assumed that there are no boundary injections (fictitious null-injection buses can be artificially created to achieve this). A decoupled estimator is used incorporating a set of linear constraints, which are introduced to force state variables in overlapping areas to assume the same values. Then, certain off-diagonal blocks are ignored in the matrices arising in the normal equations, in such a way that only boundary state variables are involved when updating the state variables within each area.

Several coordination schemes are tested: (1) apply the coupling constraint terms after every iteration of the local estimators (this algorithm actually belongs to the category of methods described in the following section); (2) first allow the local estimators to converge to the desired tolerance, and then apply the coupling constraint corrections without any further local estimation iterations; (3) allow the local estimators to converge to a tolerance relatively close to the desired one, and from then on, use an alternating iterative scheme between the local estimations and the coupling constraints corrections (this is a hybrid algorithm between those described in this and the next section). Under scheme 1, each area $\mathrm{SE}$ is performed in a synchronous manner, requiring coordination at each iteration. This requires a lot of information exchange. On the other hand, scheme 2 is of an asynchronous nature, as computations can continue even in the absence of information from other areas.

In the work of Korres and Contaxis, in 2000 [22], the entire system is divided into non-overlapping areas. Each area is individually solved, disregarding boundary measurements so that the state variables of adjacent areas do not show up. Then, at the second level, a reduced model involving tie lines and boundary measurements is handled. In this reduced model, the areas become "supernodes" linked by tie lines, while "equivalent" power flow measurements, representing the sum of power flows on the tie lines incident to each boundary bus, are used. The results of the second step are not used subsequently to update the estimates of internal nearby buses.

In [1] the use of Internet is suggested to exchange the necessary information during the coordination phase. In order to obtain phase angles of each area with respect to a global reference, phase differences across tie-lines are computed, and then the average is taken for the set of tie-lines connecting each couple of areas. The authors state that "the average scan time to get SE outputs may be of the order of 4 minutes, and therefore the Internet is well suited as the communication medium". This may not be true, however, in all cases.

All of the above methodologies provide suboptimal estimates as they imply neglecting in local SEs some nearby external and/or boundary measurements. However, for most of them, test results show acceptable accuracy in normal conditions. Besides, bad data close to boundaries, if not properly identified and removed, can degrade the accuracy of boundary and nearby variables and also, in some cases, affect convergence of the distributed SE process.

\subsection{Coordination at the iteration level}

In 1974, Kobayashi et al. [8] applied the model coordination principle of Mezarovic [34] from hierarchical system theory to the problem of state estimation. System decomposition into non-overlapping areas, connected by tie-lines, is considered. One iteration of the overall process consists of three steps: (1) using internal measurements solve local WLS problems to update internal variables, with $u$ and border state variables fixed to their most recent values; (2) using interconnection measurements solve a WLS problem for the interconnection area to update $u$, with all state variables fixed to their current values; (3) using the whole set of measurements, coordinate previous steps by computing border state variables, with $u$ and internal state variables fixed to their current values.

In 1979, Marsh and Cristi [10] formulated the WLS state estimation as an optimization problem with equality constraints. The algorithm is developed while assuming the system composed of several interconnected areas. The equality constraints come on one hand, from the power balance equations at each node, and on the other hand, from the diakoptical formulation of the interconnections between subsystems. They are taken into account by introducing Lagrange multipliers. Minimization is performed by means of a steepest descent algorithm. Each iteration requires a high amount of data transfer while proper convergence may lead to a relatively large number of iterations. Bad data analysis is not considered and appears to be a challenging problem.

In 1981, Brice and Cavin [13] developed a hierarchical distributed algorithm as follows: at each iteration, the central processor broadcasts the current value of the state vector. Then, each satellite processor computes its portion of both the Jacobian matrix and the right hand side vector of Eq. (2), which are sent back to the central processor. Next, all matrix and vector components are gathered at the central level, where the Normal equations are built and solved, and the process is repeated. Therefore, only a small fraction of the computations is parallelized, but the optimal solution is obtained in the same number of iterations as in the conventional formulation. In order to increase the amount of computations performed in parallel, the solution of the Normal equations can be also distributed among existing processors by applying a Gauss-Seidel iterative method, instead of using a direct solution approach based on Cholesky factorization.

The outstanding feature of the work by Iwamoto et al., in 1989 [17], is that rectangular coordinates, along with an extension of the popular second-order load flow algorithm, previously developed by Iwamoto and Tamura, are resorted to. The main advantage is that a constant Jacobian matrix naturally arises, significantly reducing the computational burden. In the problem formulation the interaction among subsystems is taken into account through the tie-line bus voltages. The hierarchical structure of the proposed method consists of two levels: the upper level, where the optimal tie-line bus voltages are evaluated; and the lower level, where the optimal states of each subsystem are determined by minimizing a cost function that involves the entire system. This constitutes a significant difference from a majority of published methods, first solving the local levels and then coordinating the solution at the upper level.

El-Keib et al. [20] presented in 1992 a multi-area approach for solving the Weighted Least Absolute Value (WLAV) state estimation problem. The proposal is based on the Dantzig-Wolfe Decomposition Principle, applied to the resulting LP problem. Each subproblem is solved independently by the revised simplex method and sends the solution to a master problem. The master problem incorporates this information and its solution is used to modify the objective functions of the subproblems. The process is continued till optimality is reached.

In 1995, Falcao et al. [21] proposed the three coordination schemes described in the previous section, one of them exchanging information at the iteration level.

Ebrahimian and Baldick [23] applied in 2000 the so-called "Auxiliary Problem Principle", a well-known technique in large-scale optimization, to develop a two-step procedure based on border-bus overlapping areas. The overall WLS objective function is expressed as the sum of partial functions, each one corresponding to a subsystem. Additional equality constraints are considered expressing the fact that the estimates of boundary buses in adjacent areas should be identical. The problem is decomposed by linearizing the augmented Lagrangian, yielding an iterative procedure in which subsystem solutions (involving several inner iterations) are alternated with Lagrange multiplier updates (outer loop). When solving 
each subsystem, the border information is interpreted as a set of pseudo-measurements. The optimal solution is approached as outer iterations progress, but several constants should be tuned for a particular system to guarantee convergence.

A similar approach was adopted by Aguado et al. in 2001, who also tested a Lagrangian Relaxation-based iterative scheme [24].

In general, other SE related issues, such as observability analysis and bad data handling are not fully addressed in papers dealing with MASE methods. A notable exception is the recent work [33], where distributed solutions to those problems are proposed.

\subsection{Incorporation of PMUs}

The incorporation of PMUs in a hierarchical SE process was first considered by Zhao and Abur in 2005 [25]. The resulting areas overlap over tie-lines allowing boundary measurements (injections and tie-line power flows) to be used by adjacent areas [26]. This requires transferring to the central entity the topology of those lines internally connected to the boundary buses, yielding two or more estimates for shared buses. Then, the second level receives the boundary estimates from each area, the boundary measurements (including PMUs) and the necessary values of covariance matrices corresponding to locally estimated boundary variables. Boundary variables are re-estimated along with area slack phase angles.

Yan et al., in 2006 [27], developed a decomposition procedure based on the bordered-block diagonal form (BBDF) of the gain matrix. It is assumed that PMUs provide real-time boundary states, and hence power flows between subsystems, exactly. Then, using the data of PMUs as boundary conditions, the problem of distributed WLS decoupled state estimation turns into a multiarea optimization problem with equality constraints.

In the work of Jiang et al., 2007 [29], the overall system is decomposed into a certain number of non-overlapping subsystems based on a geographical basis. Each subsystem conducts its local SE with respect to its own slack bus, disregarding boundary power injections. Also each subsystem has a PMU installed at the slack bus. Measurements from PMUs coordinate the SE solution of each subsystem. A sensitivity analysis is performed for each bus to assess the degrees of impact from the neighboring subsystems. Those buses with a sensitivity exceeding a threshold are re-estimated at the central level along with boundary buses. The central SE incorporates the following information: all tie-line power flows, boundary injections, other internal measurements related to the sensitive internal buses and estimates of both boundary and sensitive internal buses, which are considered as pseudo measurements.

Jiang et al. [31] have recently (2008) presented a diakopticbased SE algorithm. In the proposed approach, the SE problem is partitioned into a number of subproblems, obtained by removing tie-line measurements. Intermediate subsystem solutions are sent to a central computer for completing the state estimation process by taking tie-line measurements into consideration. The capability of PMUs to provide accurate synchronization of measurements is used to make each subproblem solvable and to coordinate the voltage angles of each subsystem SE solution. The optimal solution is reached.

\subsection{Factorized SE}

A different MASE perspective arises when each individual substation is regarded as an area. This allows a majority of raw measurements to be pre-processed by a linear estimator at the substation level [32]. The information provided by this stage, essentially composed of power flows and voltage magnitudes, is then integrated within the framework of a conventional SE. The main advantage of this hierarchical procedure, particularly in future substations with highly redundant measurement sets, is that the linear prefiltering phase can be run in a decoupled and geographically distributed manner, significantly reducing the bandwidth requirements. As a byproduct, a reduction in the computational effort is also achieved.

\section{Decentralized MASE}

In this category of methods the central coordinator is missing, neighboring areas directly exchanging border information with each other.

Each area proceeds in a similar fashion to the local solution phase of the hierarchical scheme. Internal measurements of area $k, z_{i k}$, can be readily used, as they are functions exclusively of state variables within the same area. On the contrary, border measurements involve state variables of neighboring areas $j$ according to:

$z_{b k}=h_{b k}\left(x_{i k}, x_{b k}, x_{b j}\right)+e\left(z_{b k}\right)$

When iteratively solving the WLS problem in area $k$, the state vector components in (6) corresponding to adjacent areas, $x_{b j}$, must be replaced by the best available estimates, $\tilde{x}_{b j}$, submitted through the bilateral communication channel. This constitutes a relaxationbased distributed implementation of the global WLS problem. The information exchange can take place in a synchronous manner, provided it is feasible and convenient for all processors to run at the same pace, or asynchronously.

Typically, decentralized procedures take longer to converge than hierarchical ones, as they neglect some of the information handled by the central processor. Furthermore, bad data detection issues are more involved.

\section{Literature survey on decentralized MASE}

Table 2 provides a classification of fully distributed MASE methods, according to the different criteria identified in Section 5.

Due to space limitations only the most representative proposals are briefly reviewed.

In 1970, in his fundamental paper [35], Schweppe briefly described two techniques to reduce the computational burden of WLS estimation. By adding a fictitious bus at the mid-point of each tie-line, the system is supposed to be composed of border-bus overlapping areas. The first technique, termed "spatial quantization" consists of performing independent local estimations using within each region the corresponding local measurements. Major drawbacks of this very preliminary idea are the following: (i) no coordination between local estimates is performed, which induces loss of accuracy in tie-line power flow estimates (one mid-tie line appears as an antenna in each area); (ii) difficulty to identify bad data on tie-lines where local redundancy is poor.

In the second technique, denoted "spatial sweep", regions are estimated sequentially using, in addition to local measurements, the estimates of fictitious buses in tie-lines of neighboring areas already estimated. Relaxation with several iterations of this sweep procedure is needed, processing regions in different orders. This iterative formulation can reduce drastically the computational efficiency.

In the work by Brice and Cavin [13], reviewed in the previous section, the authors outlined also a relaxation-based approach, by which each processor solves asynchronously the non-linear WLS problem corresponding to its area of influence. The relaxation algorithm tries to obtain the state variables that minimize the objective function of its own area under the assumption that the state variables of adjacent areas have reached the optimal values, which is not actually the case. This slows down the overall convergence, compared with the iterative solution of the Normal equations applied to the entire network, but the computations are 
Table 2

Classification of decentralized MASE methods.

\begin{tabular}{|c|c|c|c|c|c|}
\hline Reference, author & Area overl. $^{a}$ & Solut. meth. ${ }^{b}$ & Estim. state ${ }^{c}$ & Meas. type ${ }^{d}$ & Coord. scheme ${ }^{\mathrm{e}}$ \\
\hline [35], Schweppe & MO & NE-R & Sub & $\mathrm{C}$ & SE-It \\
\hline [13], Brice & NO & NE-R & Opt & $\mathrm{C}$ & It \\
\hline [36], Lin & MO & $\mathrm{O}$ & Opt & $\mathrm{C}$ & It \\
\hline$[37,38]$, Lin & MO & NE-R & Opt & $\mathrm{C}$ & It \\
\hline [39], Carvalho & MO & $\mathrm{NE}-\mathrm{H}$ & Sub & C & It \\
\hline [40], Huang & MO & NE-R & Opt & C & It \\
\hline [41], Conejo & MO & NE-R & Sub & $\mathrm{C}$ & It \\
\hline
\end{tabular}

a NO: non-overlap; MO: minimally overlap; FO: fully overlap.

b NE: Normal equations; R: relaxation; O: optimization; $\mathrm{H}$ : heuristic.

c Opt: optimal; Sub: suboptimal.

d C: conventional only; P: considers PMU.

e SE: SE level; It: iteration level.

fully distributed. There is also a need for each processor to receive the current values of nearby state variables, significantly increasing the communication requirements. Given appropriate convexity assumptions, convergence of this asynchronous process is always guaranteed, no matter in which order and at which rate each subproblem is solved. Implementation of bad data analysis on a decentralized way is difficult.

The work by Lin, in 1990 and 1992 [36,37], combines Recursive Quadratic Programming with the Dual method. The distributed $\mathrm{SE}$ is aimed at its utilization in a decentralized control system, assuming a high speed data communication network, which is topologically the same and physically in parallel with the power network (a local processor is assigned to each bus). Global convergence of the distributed scheme to the optimal solution is shown.

In a subsequent work by Lin and Lin [38], recognizing the complexity and practical difficulties of this approach, in terms of both software and hardware, the authors proposed the following modifications: (a) each processor is in charge of an entire area, not a single bus, leading to a small-scale distributed architecture interconnected by a tree-shaped communication system; (b) a much simpler partially asynchronous block-Jacobi method is adopted.

Like in the previous work, Carvalho and Barbosa, 1998 [39], assume that the computer systems of adjacent areas are connected by fast data communication links, forming a computer network. That is, there is no centralized coordinating computer. The entire system is divided into border-bus overlapping areas. Fictitious, null-injection border buses are created if necessary so that boundary injections do not appear. When updating the state variables of a given area, the values of state variables corresponding to border buses are replaced by weighted averages of those computed in neighbor areas, where the weighting coefficients are obtained from the diagonals of the inverse gain matrices. Two algorithms are considered: (1) the SEs within each area are run in a synchronous manner, information being exchanged at the iteration level. This leads to inefficiencies because of processors being idle part of the time; (2) each area fully performs the SE in an asynchronous manner, correction terms being exchanged only at the end. Some gain in computation time is obtained at the cost of accuracy deterioration. The results are anyway acceptable if redundancy is high enough.

Conejo et al. [41] proposed in 2007 a multi-area decentralized SE procedure, based on optimization concepts. The resulting algorithm, closely resembling the relaxation-based approaches of Brice-Cavin [13] and Carvalho-Barbosa [39], proceeds as follows: (1) initialize state variables for all areas; (2) each area solves its corresponding problem, using available values of boundary state variables as pseudo-measurements; (3) if state variables do not change significantly within two iterations, stop; the solution has been reached. Otherwise, neighboring areas interchange their estimates for state variables corresponding to border buses, and the procedure continues in (2). No indication is provided on how to select appropriate weights for border pseudo-measurements.

\section{Conclusion}

Multi-area state estimation methods were introduced nearly forty years ago, partly to circumvent the limitations of by then available computers. The tremendous increase in computational power has not decreased the attractiveness of MASE. On the contrary, it has got renewed interest owing to the need of properly monitoring energy transactions across TSO borders in large interconnections, while at the same time processing the real-time data at the most appropriate place, and possibly preserving their confidentiality. The expected increase of smart grid applications also calls for the MASE techniques, in order to prevent communication infrastructures from being unnecessarily burdened with the resulting information explosion.

Over thirty references on MASE, including both journals and conference proceedings, are analyzed in this work. As a consequence, a taxonomy of MASE is established with the help of previously identified classification criteria. Such criteria include: area overlapping degree, computer architecture, coordination scheme, process and measurement synchronization and solution methodology. For each major category of methods (hierarchical and decentralized) an effort has been made to identify the relevant state and measurement vector components, as well as their interactions during the solution phases, all this under a common notation. Then, a brief presentation is made of a relatively large selection of references, trying to point out their distinguishing features and limitations.

A somewhat expected but noteworthy conclusion of this work is that there exists a compromise solution between optimality and computational cost (or complexity) of the resulting procedure. A majority of hierarchical procedures lead to suboptimal, yet accurate enough solutions in just two steps, particularly in the presence of PMUs, which increase the linearity of the resulting models. Fully distributed schemes tend to be simpler, at the cost of poorer convergence to the optimal solution.

\section{Acknowledgements}

This work was performed in the context of the PEGASE project funded by European Community's 7th Framework Programme (grant agreement No. 211407). The Spanish authors also acknowledge the support of the Spanish DGI under grants ENE 2007-62997 and ENE 2010-18867.

\section{References}

[1] A.R. Khatib, L. Mili, A. Phadke, J. De La Ree, Y. Liu, Internet-based wide-are information sharing and its roles in power system state estimation, in: IEEE-PES Winter Power Meeting, Columbus, Ohio, January 28-February 1, 2001.

[2] www.coreso.eu.

[3] T. Van Cutsem, M. Ribbens-Pavella, Critical Survey of hierarchical methods for state estimation of electric power systems, IEEE Trans. Power Apparatus Syst. PAS-102 (October (10)) (1983) 3415-3424. 
[4] M. Shahidehpour, Y. Wang, Communication and Control in Electric Power Systems: Applications of Parallel and Distributed Processing, Wiley-IEEE Press, July 2003.

[5] A. Abur, A. Gómez Expósito, Power System State Estimation: Theory and Implementation, Marcel Dekker, April 2004.

[6] A. Monticelli, A. Garcia, Reliable bad data processing for real-time state estimation, IEEE Trans. Power Apparatus Syst. PAS-102 (May (5)) (1983) 1126-1139.

[7] K.A. Clements, O.J. Denison, R.J. Ringlee, A multi-area approach to state estimation in power system networks, in: IEEE PES Summer Meeting, Paper C72 465-3, San Francisco, CA, July 1972.

[8] H. Kobayashi, S. Narita, M.S.A.A. Hamman, Model coordination method applied to power system control and estimation problems, in: Proc. of the IFAC/IFIP 4th Int. Conf. on Digital Computer Appl. to Process Control, 1974, pp. 114-128.

[9] M.R. Irving, M.J.M. Sterling, Multi-level state estimation and optimal control in: Proc. of the IFAC Symp. on Comp. Applic. in Large-Scale Power Syst, New Delhi, August 1979, pp. 117-125.

[10] J.F. Marsh, R. Cristi, State estimation on electric power systems using partitioned network models, in: Proc. of the IFAC Symp. on Comp. Applic. in Large-Scale Power Syst, New Delhi, August 1979, pp. 97-103.

[11] T. Van Cutsem, J.-L. Horward, M. Ribbens-Pavella, A two-level static state estimator for electric power systems, IEEE Trans. Power Apparatus Syst. PAS-100 (August (8)) (1981) 3722-3732.

[12] Y. Wallach, E. Handschin, C. Bongers, An efficient parallel processing method for power system state estimation, IEEE Trans. Power Apparatus Syst. PAS-100 (November (11)) (1981) 4402-4406.

[13] C.W. Brice, R.K. Cavin, Multiprocessor static state estimation, IEEE Trans. Power Apparatus Syst. PAS-101 (February (2)) (1982) 302-308.

[14] K. Seidu, H. Mukai, Parallel multi-area state estimation, IEEE Trans. Power Apparatus Syst. 104 (May (5)) (1985) 1025-1034.

[15] M.S. Kurzin, Real-time state estimation for large-scale power systems, IEEE Trans. Power Apparatus Syst. PAS-102 (July (7)) (1983) 255-263.

[16] K.L. Lo, M.M. Salem, R.D. McColl, A.M. Moffatt, Two-level state estimation for large power system. I. Algorithms and II. Computational experience, IEE Proc.Gener. Transm. Distrib. Part C 135 (July (4)) (1988) 299-318.

[17] S. Iwamoto, M. Kusano, V.H. Quintana, Hierarchical state estimation using a fast rectangular-coordinate method, IEEE Trans. Power Syst. 4 (August (3)) (1989) 870-879.

[18] A.A. El-Keib, C.C. Carroll, H. Singh, D.J. Maratukulam, Parallel state estimation in power systems, in: Proc. 22nd Southeastern Symposium on System Theory, CS Press, Los Alamitos, CA, Catalog No. 2038, March 1990, pp. 255-260.

[19] S.S. Ahmed, A. Brameller, New algorithm for diakoptical static state estimation, IEE Proc. Gener. Transm. Distrib., IEE Proc. C 138 (May (3)) (1991) 185-192.

[20] A.A. El-Keib, J. Nieplocha, H. Singh, D.J. Maratukulam, A decomposed state estimation technique suitable for parallel processor implementation, IEEE Trans. Power Syst. 7 (August (3)) (1992) 1088-1097.

[21] D.M. Falcao, F.F. Wu, L. Murphy, Parallel and distributed state estimation, IEEE Trans. Power Syst. 10 (May (2)) (1995) 724-730.

[22] G.N. Korres, G.C. Contaxis, Application of a reduced model to a distributed state estimator, IEEE Power Eng. Soc. Winter Meet. 2 (2000) 999-1004.
[23] R. Ebrahimian, R. Baldick, State estimation distributed processing, IEEE Trans. Power Syst. 15 (November (4)) (2000) 1240-1246.

[24] J.A. Aguado, C. Perez-Molina, V.H. Quintana, Decentralised power system state estimation: a decomposition-coordination approach, IEEE Porto Power Tech. Proc. 3 (September) (2001) 6.

[25] L. Zhao, A. Abur, Multiarea state estimation using synchronized phasor measurements, IEEE Trans. Power Syst. 20 (May (2)) (2005) 611-617.

[26] A. Abur, Distributed state estimation for mega grids, in: Proc. of the 15th PSCC Liege, August 2006, pp. 22-26.

[27] L. Yan, Z. Xiaoxin, Z. Jingyang, A new algorithm for distributed power system state estimation based on PMUs, in: International Conference on Power System Technology, PowerCon 2006, October 2006.

[28] R.F.Jeffers, Techniques for wide-area state estimation in power systems, Master Thesis, Virginia Polytechnic Institute, November 2006

[29] W. Jiang, V. Vittal, G.T. Heydt, A distributed state estimator utilizing synchronized phasor measurements, IEEE Trans. Power Syst. 22 (May (2)) (2007) 563-571.

[30] M.Y. Patel, A.A. Girgis, Two-level state estimation for multi-area power system, in: IEEE Power Engineering Society General Meeting, June 2007.

[31] W. Jiang, V. Vittal, G.T. Heydt, Diakoptic state estimation using phasor measurement units, IEEE Trans. Power Syst. 23 (November (4)) (2008) 1580-1589.

[32] A. Gómez Expósito, A. de la Villa Jaén, Two-level state estimation with local measurement pre-processing, IEEE Trans. Power Syst. 24 (May (2)) (2009) 676-684.

[33] G.N. Korres, A distributed multiarea state estimation, IEEE Trans. Power Syst., 10.1109/TPWRS.2010.2047030.

[34] M.D. Mezarovic, D. Macko, Y. Takahara, Theory of Hierarchical Multilevel Systems, Academic Press, 1970.

[35] F.C. Schweppe, J. Wildes, D.B. Rom, Power system static state estimation, Part I, II, III, IEEE Trans. Power Apparatus Syst. PAS-89 (January (1)) (1970) $120-135$.

[36] S.Y. Lin, A method to solve power system static-state estimation problem by using a network of processors, in: Proc. 29th CDC, Hawaii, Honolulu, December 5-7, 1990, pp. 3067-3072.

[37] S.Y. Lin, A distributed state estimator for electric power systems, IEEE Trans. Power Syst. 7 (May (2)) (1992) 551-557.

[38] S.-Y. Lin, C.-H. Lin, An implementable distributed state estimator and distributed bad data processing schemes for electric power systems, IEEE Trans. Power Syst. 9 (August (3)) (1994) 1277-1284.

[39] J.B. Carvalho, F.M. Barbosa, Parallel and distributed processing in state estimation of power system energy, in: Electrotechnical Conference, 1998 MELECON 98, 9th Mediterranean, vol. 2, May 18-20, 1998, pp. 969-973.

[40] G.M. Huang, J. Lei, A concurrent non-recursive textured algorithm for distributed multi-utility state estimation, in: Power Engineering Society Summer Meeting, 2002 IEEE, vol. 3, July 2002, pp. 1570-1575.

[41] A.J. Conejo, S. de la Torre, M. Canas, An optimization approach to multiarea state estimation, IEEE Trans. Power Syst. 22 (February (1)) (2007) 213-221. 